\title{
Posgrado en Artes, un esfuerzo por entender al sujeto desde la transdisciplinariedad
}

\author{
Dra. Thelma Itzel Ramírez Cuervo \\ thelmacuervo@yahoo.com \\ Dr. Jaime Torija Aguilar \\ jaime_torija@hotmail.com \\ Benemérita Universidad Autónoma de Puebla
}

\begin{abstract}
Resumen. Este trabajo tiene como punto de partida la creación de un plan de estudios que se basa en la transdisciplinariedad y los cambios de paradigma que esta visión implica. Mientras se describen los desarrollos científicos y tecnológicos se hace un recuento histórico que delata la prioridad dada al objeto de conocimiento, sobre todo en la modernidad. Posteriormente, se abordan las perspectivas filosóficas, el contexto del programa y la institución que lo alberga, así como los fundamentos pedagógicos. El posgrado se ubica en el paradigma de la complejidad; lo que justifica la presencia del sujeto como co-creador del conocimiento. La maestría en Artes: inter y transdisciplinariedad de la Universidad Autónoma de Puebla, México, pretende el encuentro de artistas a la formación de una identidad singular y múltiple donde el concepto de arte se actualiza día con día.
\end{abstract}

Palabras clave: Artes, Transdisciplinariedad, Sujeto.

\section{Introducción}

El dos de agosto de 2016 dieron inicio los cursos del posgrado Maestría en Artes: inter y transdisciplinariedad de la Facultad de Artes de la Benemérita Universidad Autónoma de Puebla (BUAP). Dicho proyecto significa un reto no sólo por los siete años que tardó en ser aprobado, sino por las múltiples modificaciones acaecidas desde su proyección original. Al principio se planteó como un posgrado en composición electro acústica, pasando por un posgrado en enseñanza de las artes, hasta la elaboración de un proyecto que nos permitiera -a través del arteaproximarnos a los fundamentos de aquello que nos hace humanos; esto es, los procesos creativos, la comunicación, y la elaboración de redes de pensamiento que tendieran a la resolución de problemas integrando perspectivas y metodologías afines que desembocaran en áreas donde las artes tienen posibilidades de reconocimiento y competitividad en el ámbito profesional: gestión, educación, creación e investigación. El tiempo de práctica, aunque corto, ha permitido vislumbrar algunas problemáticas que rebasan la esfera de lo académico.

Para llevar a cabo ese proyecto nos posicionamos en la transdisciplinariedad porque permite la apertura de un nuevo paradigma que, entre otras cosas, se interesa e incluye los momentos subjetivos de los individuos; por lo tanto, nos inclinamos hacia una perspectiva fenomenológica. El concepto de transdisciplinariedad no es desconocido, aunque se diversifica por su uso en los últimos tiempos. En el ámbito académico se observa que después de la 
mitad del siglo XX diversos especialistas, tanto de las ciencias exactas como de las humanidades, incluyen nuevos conceptos en torno a la misma concepción en los procesos de investigación como son multidisciplinariedad, interdisciplinariedad, ciencia de la complejidad o teoría del caos. Nociones que se dirigen a transgredir los límites de las diversas disciplinas que tradicionalmente están localizadas en la ciencia. Al unir el prefijo trans-, que significa "más allá" o "a través", con el concepto de disciplina se advierte inmediatamente dicha transgresión. En pocas palabras, se puede ver a la transdisciplinariedad como el camino que nos induce al rompimiento de los paradigmas tradicionales.

Lo anterior significa que la concepción de la ciencia (el todo), que en la actualidad se conforma por diversas especialidades (las partes) y con base en un racionalismo riguroso, está siendo cuestionada en sus estructuras básicas como son la univocidad, la linealidad y la simplicidad. En contraposición, el sostén de la transdisciplina se encuentra en la multiplicidad, la diversidad y en "su carácter inacabado, en construcción y por ello, de indeterminado y también construible" (Espina, 2004, p. 11).

La academia trata de dejar atrás los métodos clásicos que rigen a la ciencia y que se circunscriben a los esquemas de la lógica y de la racionalidad; también busca distanciarse de la dirección "omnicomprensiva" y de la visión unívoca de la realidad (Espina, 2004). Ante dicha visión se reconoce el colapso con los lineamientos parcelarios del conocimiento. Por lo tanto, la transdisciplina se nutre de enfoques múltiples, entre los que resaltan la complejidad, el constructivismo, la hermenéutica y el pensamiento sistémico, donde el conocimiento no se construye en la separación.
Adentrarnos en el paradigma de la transdisciplina nos induce, necesariamente, a detenernos en una reflexión sobre el sujeto, pues al ser el centro de donde se origina el conocimiento (inducido por la tradición filosófica occidental), paradójicamente sufre un drástico desplazamiento por la ciencia determinista, la filosofía estructuralista y positivista (Morin, 2014, p. 81).

\section{Algo de historia}

Para posicionarnos en el paradigma de la transdisciplinariedad creemos importante mencionar de forma muy general la importancia del sujeto en algunos periodos anteriores a la Modernidad, porque nos permite reconocer los cimientos del conocimiento en las diferentes etapas de la historia. El cuestionamiento de su inclusión o exclusión tiene sus orígenes en la Antigüedad. Un ejemplo claro es la polémica intensa suscitada por la existencia o no del vacío entre la corriente atomista (Demócrito, Epicuro, Lucrecio), que reconoce la vacuidad, y aquellos que la niegan como Parménides, Platón, Aristóteles o los Estoicos. En esta oposición está en juego el concepto del sujeto.

En este periodo se advertían las inconsistencias del sujeto desde el momento en que se discutía la existencia de la nada, argumentando que lo que no existe no es objetivo. Sólo se constata lo que se ve; lo invisible estará fuera del rango de la verdad. Para algunos filósofos dicha postura (creer en el vacío) significó un alejamiento del camino de la realidad, ya que ésta exige elementos visibles que sólo se muestran a través de la existencia del ser. Por lo tanto, negar la presencia del vacío, según nuestra perspectiva, induce a desprenderse de las opiniones del sujeto. Esta argumentación se basa específicamente en la idea de calificar a los pensadores creyentes de la vacuidad como individuos 
que sostienen ideas de cosas inexistentes, pues la base que sostiene dicha irrealidad está sustentada por la metafísica y, por consiguiente, por el sujeto que guarda lo simbólico y metafórico.

En la Edad Media existió una tendencia desmedida hacia las expresiones del sujeto, en la cual se revelan de forma nítida los valores y principios morales que forman parte de las necesidades de lo humano, como una forma de encontrar respuestas a los fenómenos de la naturaleza, a las interrogantes teológicas, a las relaciones políticas y sociales. Aquí, la presencia o ausencia del sujeto en el conocimiento de la realidad se desplaza hacia las propuestas filosóficas que ofrecen la escolástica y el nominalismo, tendencias basadas en las manifestaciones teológicas. En este periodo, el sujeto es el principal interlocutor, el que menciona, el que formula; por consiguiente, el que le da existencia a las cosas (Beuchot, 2002, p. 26). En conclusión, en el medioevo hay una visión más profunda del sujeto a través del camino de la metafísica, la cual será alimentada fundamentalmente por las creencias en la divinidad cristiana, lo que influye para hacer una introspección hacia la "centralidad del hombre" (Vattimo 2007, p. 37); es decir, hacia el sujeto. Es en la Modernidad cuando nuevamente se excluye al sujeto de la percepción de la verdad o, más bien, de la veracidad. Se ha dicho que Descartes orienta con sus reflexiones este distanciamiento. Es, entonces, desde el momento en que el razonamiento se basa en las matemáticas que se concibe una realidad totalmente determinada.

Dicha situación será aprovechada por el hombre para lograr imponer la medida y la norma a todo ente; asímismo, le permitirá desarrollar, en un alarde de concebirse como la medida de todas las cosas y como dominador del mundo, el poder ilimitado del cálculo. El mecanismo para asegurar y ajustar la teoría se llevará a cabo a través de la técnica que se impondrá en un proceso ascendente con el fin de hacerse necesaria, objetivar lo vivo -la tierra, la naturaleza- y, al mismo tiempo, para consolidar la condición de sujeto en el hombre.

En la Modernidad, la técnica es una forma de des-ocultar, de producir, y no de manipular, confeccionar o de "aplicar medios" (Heidegger, 1997, p. 122). Este "desocultamiento" es la labor sustancial que la técnica moderna realiza para llevar a cabo una liberación de la energía $\mathrm{y}$, de esta forma, explotar a la naturaleza (Heidegger, p. 1997). Se acabó, pues, el hecho de lograr una finalidad eficiente y "dar lugar a"; todo el potencial oculto de la naturaleza es "desocultado" para conducirla a una transformación, y se da un sometimiento de la misma para llegar a ser objeto del hombre. Desocultar le da a la técnica un papel prepotente que permite objetivarse sobre la tierra y, al mismo tiempo, distanciar al hombre del descubrimiento que ejercía sobre ella; es decir, hay un desapego de lo humano.

La producción que en algún momento fue deliberada e intencionada por el hombre, ahora escapa de sus manos: ya no puede expresarse de manera individualizada, sino que se ha introducido en la esencia misma del hombre como sociedad. El objeto exige, por lo tanto, una especial consideración sin la intromisión del sujeto, ya que su permisibilidad frenaría el desbordante papel de la técnica.

Cuando en la Modernidad se afirma al individuo como ser racional, garante de la subjetividad y centro del universo que adquiere autonomía, derechos, responsabilidades, y que asume un componente propio que lo diferencia del otro y lo hace único (persona libre en los actos que le dicta la razón), se establece des- 
de la ciencia moderna un distanciamiento radical que anula completamente al sujeto del objeto de conocimiento por ser una "interferencia" en la objetividad del dato. Su eliminación responde, entre otras causas, al sometimiento de los datos obtenidos a un proceso mecánico de causa y efecto, como una fórmula reduccionista, circunscribiéndose exclusivamente a lo que se dice qué es, sin considerar las impresiones de quién conoce -efecto llevado a cabo, también por el desarrollo de la ciencia y la tecnología, en donde la técnica supera los fines buscados por el hombre-. Se ha separado el sujeto del objeto con el argumento de atribuirle a la ciencia el patrimonio de dominar la verdad como valor de objetividad. La justificación, afirma Mayra Espina (2004), se basa en separar

(...) tajantemente la realidad objetiva (como dimensión externa) del sujeto que la conoce y esto es lo que sustenta la acción de conocer: la conciencia de esa separación, que permite observar, medir, clasificar, algo que está fuera del sujeto y suficientemente alejado de él para evitar cualquier interferencia al 'captar', 'descubrir', las cualidades de la realidad objetiva, encontrar las leyes propias de la realidad estudiada ( $\mathrm{p}$. 19).

Dichas oscilaciones son motivadas por una visión de verdad, la cual, por un lado, trata de instituirse a través de una realidad lineal, continua y única que se fundamenta en una lógica inductiva tendiente a establecer leyes generales basadas específicamente en el objeto; por otra parte, reconoce que dicha verdad (con base en la verificación empírica y lógica) no tiene "un fundamento cierto para el conocimiento y de que éste contiene sombras, zonas ciegas, agujeros negros" (Morin, 2010, pp. 24-25); por lo tanto, el actual conocimiento no puede crear certidumbre en los fundamentos que se plantea, pues requiere necesariamente de los componentes del sujeto (emociones, imaginación, intuición, sueños, etc.) que impulsan elementos positivos pese a relativizar e historizar el conocimiento.

Por lo anterior, desde el siglo XX se escuchan más voces que cuestionan la exclusión del sujeto porque el método del actual procedimiento de la ciencia ha alejado al conocimiento de elementos indicativos que nutren y enriquecen la realidad o las diversas realidades. El reduccionismo, producto del proceso de simplificación racionalista, no ha tomado en consideración la entropía que se manifiesta en todos los procesos de búsqueda científica; ha desechado fenómenos de la realidad, preponderado la verificación y la lógica abanderada por la razón; es decir, ha ignorado la parte no utilizable de los elementos que interfieren cuando el sujeto lleva a cabo investigaciones en esferas como la religión, la metafísica, la interpretación, la historia personal o social, entre otras.

Del mismo modo, también es importante concebir la realidad desde una perspectiva diferente a la que tradicionalmente se ha impuesto, lo que nos lleva a pensar en "(...) una Realidad multidimensional, estructurada en múltiples niveles, que remplaza la Realidad unidimensional de un solo nivel en el pensamiento clásico" (Nicolescu, 1996, p. 40); es decir, en una realidad que es verdadera, sostenida -contraria a la concebida por las ideas filosóficas de antiguos y modernos- desde el marco de referencia del actor social. Es, quizás, esta propuesta ya recurrente desde la transdisciplinariedad, un nuevo reposicionamiento para encontrar la verdad en los diferentes niveles de la realidad, con el objeto de proyectar lo que es desde otra dimensión. En la Modernidad, las concepciones que se tienen de la ver- 
dad han llevado a tener en cuenta nuevas consideraciones: debates entre idealistas y racionalistas han enriquecido el criterio de lo que es el ser desde otras dimensiones, siendo éstas las que ofrecen una nueva realidad. Es desde esta visión que en las últimas décadas salta constantemente en los círculos científicos la necesidad de incluir en los resultados del conocimiento la propuesta del sujeto.

La transdisciplinariedad implica un gran esfuerzo porque rompe con los usos y costumbres de la ciencia reduccionista que exige la exactitud; aquí nos enfrentamos con la incertidumbre.

El proyecto de maestría intentó responder al cuestionamiento ¿Qué es el arte?, del que se derivan las siguientes preguntas: ¿qué manifestaciones o representaciones hemos elegido al concebir el arte?, ¿quién es el sujeto que las detenta?, ¿por qué el arte debe o puede manejarse como algo externo, separado -una vez más- del individuo?, ¿qué distingue a los individuos para asumirse o no como artistas? Estos planteamientos se encuentran directamente relacionados con el principio de identidad, de no contradicción y del tercero excluido, de manera que el arte se coloca dentro de la lógica disciplinar donde una cosa es lo que es y no puede, al mismo tiempo, ser otra o no ser la cosa.

Todo arte debe pasar por una técnica y método que lo valide y le permita construir reglas, objetos de medición, valor y competencia; sin embargo, vale la pena reflexionar nuevamente sobre las definiciones de técnica, método y sistema.

En el posgrado se pretende que cada estudiante reflexione sobre la base sistémica que propone la institución (entiéndase el sistema como una totalidad que integra no sólo diferentes perspectivas de las artes y otras disciplinas, sino que las asume como formas de vida). Por lo tanto, el alumno se apropia de métodos y técnicas entendidas como camino y procedimientos respectivamente. El sistema, además, no puede considerarse sin contextualización y retroalimentación de/y con otros sistemas, lo que le otorga dinamismo, noción que pareciera contraria a lo estático de la especialización.

El proceso de creación es entendido como un sistema que va más allá de la dialéctica (sus contrarios y síntesis); se concibe como un movimiento que no sólo trabaja en espirales de evolución ascendente y lineal, sino en la forma expansiva de una galaxia que integra todo a su paso, reflejándose en las diferentes disciplinas $\mathrm{y}$ dimensiones de la realidad en la que interviene el sujeto con sus circunstancias. De esta manera, el arte se piensa no sólo como mecanismo o herramienta para construir y comunicar representaciones, sino como un organismo y un sistema autopoiético.

Pongamos como ejemplo dos de los anteproyectos aceptados: el arte de crear prótesis tridimensionales en cuya hechura no sólo interviene la biología, medicina y la ciencia, sino las artes plásticas; o bien, la posibilidad de una cartografía que nos muestre los parámetros de felicidad en las distintas zonas de la ciudad y, a partir de ello, detectar problemas y resoluciones. Este último trabajo emplea estadística e historia, así como reflexiones sociológicas, filosóficas y psicológicas, además de contar con elementos plásticos, escénicos y sonoros para su composición.

\section{Contexto}

La formación del Posgrado en Artes respondió a los fundamentos establecidos en el Modelo Educativo de la BUAP, Modelo Universitario Minerva (MUM), en cuyo ideario se conjunta un enfoque constructivista con orientación sociocultural y de humanismo crítico. ¿Cómo deben asumirse los enfoques propuestos 
en el MUM para la conformación del posgrado y su ejercicio? De inicio debía corresponderse con un ideario institucional que promoviese la autorrealización del ser humano concebido éste desde una perspectiva holística.

El proyecto no pretendió conformar un diseño curricular aunque de suyo, la apertura de cualquier plan educativo exige una serie de estudios a tomarse en cuenta: estudios de pertinencia y factibilidad, así como posibilidades de vinculación que contextualizan, sustentan y fundamentan al posgrado, mismo que, inserto ya dentro de una Institución con modelo específico, sirve como herramienta de enlace, no sólo entre las distintas disciplinas que lo conforman sino al sensibilizar y desarrollar la comunicación inter e intra personal del individuo.

El enfoque constructivista en el MUM toma como base las teorías de Jean Piaget, Lev Vigotsky y Paul Ausubel, entre otros, y busca un aprendizaje significativo que se da en la interacción social. "El conocimiento se construye sobre problemas específicos, problemas sociales que se experimentan, se sistematizan, discuten y resuelven más allá del aula con sentido crítico" (2007, p. 45). En este aspecto, la estrategia didáctica del posgrado no ha sido partir de temas de investigación, sino de problemas específicos que, una vez identificados, motiven a la investigación, gestión, creación y educación como partes o momentos indispensables a seguir en cada proceso que, de esta forma, se aborda como un sistema del cual ya hemos hablado.

En el MUM la posibilidad crítica no sólo está relacionada con el humanismo, sino con el paradigma socio-crítico tendiente a la acción, la práctica y la toma de decisiones. Esto con el fin de que sus criterios de calidad se basen en la intersubjetividad y validez consensuada, de manera que el conocimiento sea construido y divergente, y que permita a los estudiantes identificar posibilidades de cambio, toda vez que se ha realizado un análisis de la realidad. Todos esos elementos que Ruiz Ruiz (2005) considera como característicos de ese paradigma repercuten en el MUM, en la insistencia del aprendizaje ligado a práctica, incluyendo la interacción multidimensional entre estudiantes, profesores y contenidos. Así, en un primer análisis de políticas y tendencias dominantes en la educación, el MUM establece:

Sería deseable la construcción de modelos educativo-académicos que, teniendo en cuenta críticamente las tendencias contemporáneas, contribuya a disminuir y revertir sus efectos nocivos - desigualdad, inequidad y ecocidio planetario - esto es, por un lado, generar alternativas de desarrollo y transformación de la sociedad $\mathrm{y}$, por otro, formar profesionales $\mathrm{e}$ investigadores críticos/as, creativos/ as y comprometidos/as con proyectos históricos, personales y colectivos, en un contexto internacional, nacional y regional (2007, p. 12).

\section{Enfoque actual. Disolución del sujeto}

La disolución del sujeto o de una idea del sujeto que ha depositado su confianza en el progreso y en el conocimiento racional, traducible a fórmulas o procesos que lo vuelven objetivo y asequible para todos, tiene que ver con el proyecto de Modernidad y con el pensamiento positivista que influye hasta nuestros días en gran parte del mundo occidental. Sin embargo, en la vida diaria nos daremos cuenta que esto no es así. Como ejemplo hemos de usar la dramaturgia o el carácter de lo dramático basado en el conflicto, la acción y el al- 
cance de objetivos. En la simetría, como rasgo connatural a la vida y lo dramático, no es posible la existencia de un personaje sin una alternancia, generalmente opuesta, que se evidencia en un cambio de situación que lo transforma; así, el sujeto (o personaje) se muestra como un plato de la balanza que se sostiene por la existencia proporcional del conflicto. En el drama, es requisito que el sujeto exista y se transforme. Su desplazamiento -la acción- es el fundamento del drama como teatro. La acción para realizarse ha de requerir una presencia o un carácter; más aún, la acción, para ser interpretada como tal, debe ser identificable para otros.

El drama no sólo se compone de acciones miméticas, también está estructurado por acciones totalmente lógicas capaces de determinar y circunscribir el entendimiento racional del que observa el acto, condicionando el teatro a una realidad que bien puede ser la realidad de lo que ocurre afuera, la realidad del día a día, la realidad social (Valles 2015, p. 52).

Rànciere, citado por Mizrahi (2015), propone: "lo real debe ser ficcionado para poder ser pensado" y esto se debe a que al cuestionar al sujeto cuestionamos el estatuto de realidad, entendida como un referente colectivo que no debe confundirse con lo real, lo realista, lo ficcional y la ilusión.

La realidad, al menos en el pensamiento europeo que nos concierne, determina presencia e identidad como necesarias para la comprensión del mundo, la generación de conocimiento, la trascendencia y la delimitación del arte. Esto lo hará a través de representaciones, las cuales, para existir, requieren de una organización artificiosa, lógica, delimitada por unidades espacio temporales. En lo que concierne al tiempo, se concibe como el tiempo lineal que se estructura y organiza mediante acciones y cambios de situación guardando una coherencia. El espacio-tiempo consta de una duración establecida que permite apenas algo semejante a una narración. Así, la narratividad subyace no sólo en las artes, sino en la vida misma y en el ejercicio que cada persona realiza para visualizar su existencia organizando las relaciones de causa-efecto que le otorgarán sentido.

Lo real será aquello que resquebraja la ilusión en cualquiera de sus niveles, aquello que provoca la experiencia amarga en el ámbito literario, y el conocimiento científico en el ámbito sociológico. Pero lo real escapa a la representación: toda representación lo es siempre de una ilusión, más o menos compartida, a la que denominamos realidad (Sánchez, 2013, p. 46).

Por más que intentemos la justificación de una existencia, el ser humano no llega al mundo con un plan hecho de antemano, ni un objetivo específico a alcanzar. Al me-

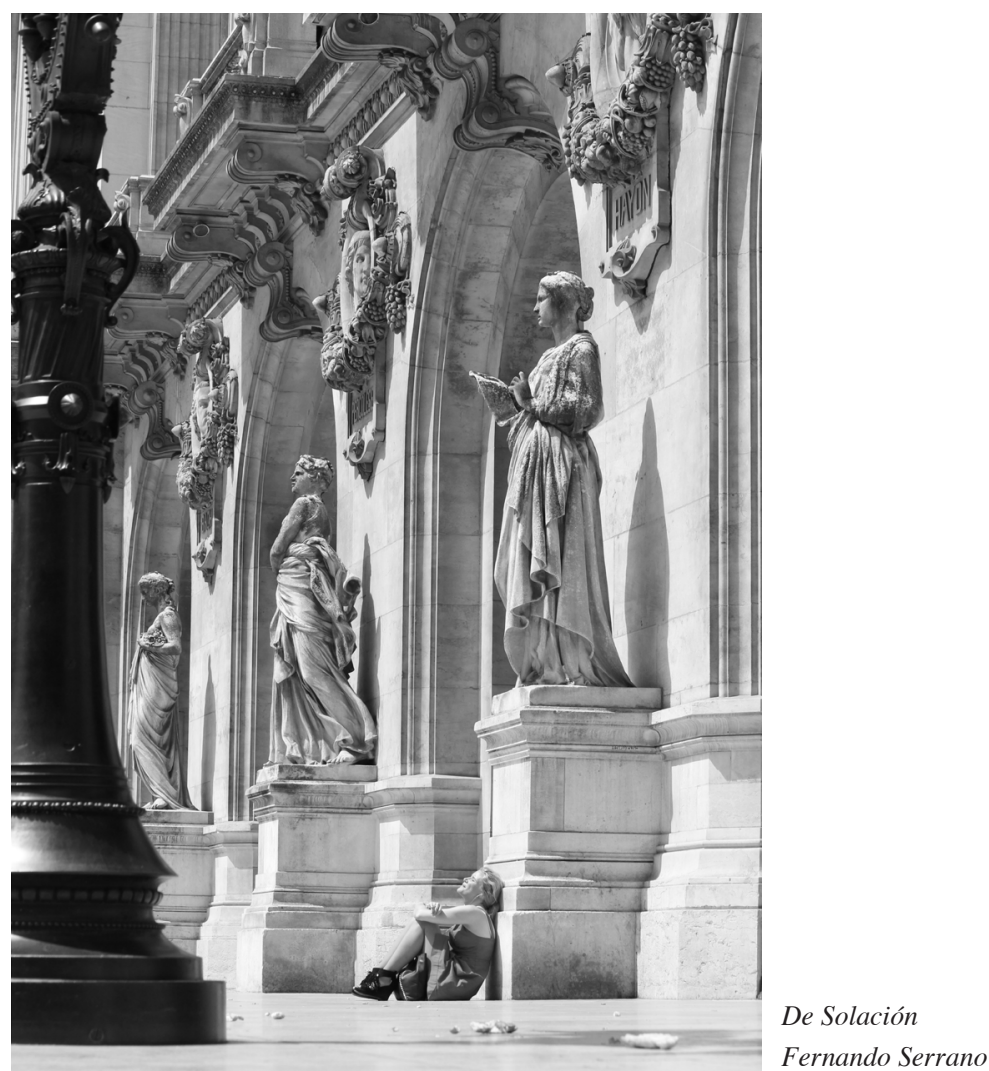


nos, aún no nos es dado reconocerlo en el plano bidimensional en que nos desarrollamos.

Ampliadas las nociones del espacio-tiempo por la multiplicidad que proveen los medios tecnológicos y posibilidades otorgadas por la virtualidad, se aspira a que cada proyecto pueda formular su propia metodología como una vía posible de aprehensión, donde la trascendencia no se finca en el cultivo de la identidad, sino en la deconstrucción de conceptos, esquemas propios y el flujo permanente de la creación en el presente. Este cambio de paradigma incide, además, en la pedagogía. La relación inmediata se establece entre los sujetos-objetos, los pormenores de una comunicación de redes, entre sistemas y modos de configurar la realidad. Somos sujetos y objetos de estudio en forma recíproca.

El tipo de estudiante que requerimos puede ser el cualquiera al que alude Sánchez (2016): un cualquiera que es capaz de asumir los riesgos de la creación conjunta, que no requiere la mecanización y la técnica del virtuosismo, ni representa la singularidad de modelos que no son accesibles para todos. La posibilidad de ese cualquiera que forma multitud, multiplicidad y probabilidades de cambio, al final de la jornada nos traería de vuelta a la existencia, siendo ésta el valor supremo que trasciende la frontera de estereotipos culturales.

El actor se vacía de sí para convertirse no en un personaje con identidad definida, sino en "uno cualquiera". Y cuando esto se hace no por virtuosismo, sino para hacer valer un discurso silenciado, este sacrificio adquiere una dimensión ética. La ética sólo existe en relación, en relación con los otros, y los otros en este caso son tanto los espectadores como aquellos que ad- quieren representación en el vaciamiento del actor (Sánchez, 2016, p. 122).

En el posgrado, la misión radica en abrazar el paradigma de la complejidad como eje rector de las propuestas o proyecciones de cada alumno. Abrirse a las posibilidades de: 1) El concepto de arte como algo no acabado o estable que se construye en el día a día; esto implica al sujeto, a la naturaleza de "lo humano" y sus contextos. 2) Elaborar proyectos sin que estrictamente haya una comprobación o hipótesis favorables. Si bien todo proyecto debe acompañarse de una investigación y reflexión profunda que pueda, incluso, sentar las bases para nuevas teorías, ello no obliga a que las hipótesis, al momento de ser verificadas, obtengan los resultados previstos. El error es posible puesto que las perspectivas que se ponen en juego permiten ampliar el rango de visión y yuxtaposición de metodologías en donde los resultados no serán igual de productivos, eficientes y verificables para todos. Aquí se manifiesta, al mismo tiempo, como prioritario, la búsqueda de sentido $\mathrm{y}$, por consiguiente, algo muy importante como objetivo de este posgrado: la construcción del objeto.

Esta construcción se hace posible durante el proceso mismo de investigación, mediante el intercambio en el campo mismo de la intervención, es decir, en el contexto de producción de conocimientos. En esta situación se abren paso las prácticas de reflexión y de cuestionamiento permanente (Araujo y Fernández, 1999, p. 245).

En esta exploración que intenta ir más allá de la comunicación pretendidamente asertiva, la disolución del sujeto es una impronta que contradice la lógica clásica. Para Valles (2015), se trata, en el traba- 
jo escénico, de la llegada a una suerte de pre-presencia; esto es, un estar antes de ser que remite al proceso de vía negativa establecido por Grotowski, donde la preparación y/o entrenamiento actoral no se observan como la adquisición y dominio de una serie de técnicas para el desarrollo de habilidades, sino una liberación para erradicar los estereotipos, clichés o formalidades aprendidas en sociedad y que se acumulan en pensamiento y cuerpo a modo de resistencias; se trata, pues, de construir una identidad montada sobre la presencia inédita del estar. También nos conduce al pensamiento de Artaud al ponerse de manifiesto la importancia del cuerpo y la encarnación como elementos indispensables para la existencia del juego estético, ético y político que representa el artista. El artista/el arte es lo que está, no el que es. Es el lenguaje, no el cuento. Esta postura implica una renovación constante, una actitud de tránsito, posibilidades múltiples que anulan el principio de identidad o de casamiento con una sola forma o estilo. "La adscripción de artistas a una determinada tradición -dice Sánchez (2016)- no puede ser definitiva, las tradiciones son movimientos, no algo estanco que da lugar a modelos puros" (p. 37); implica, sobre todo, una ética que sólo puede darse en la práctica, en lo corpóreo que atiende a la relación con los otros y con el contexto y que, por lo tanto, es una forma de práctica artística:

La ética en cuanto aquello que se activa en la toma de decisiones y se manifiesta en los hábitos de conducta sólo se da en la práctica y en la acción. Si la ética se da en la práctica, sólo cabe hablar de la ética en relación con los otros, en actuar para los otros, o al menos condicionado por los otros. El bien y el mal no pueden ser entendidos como universales absolutos [...] sino como términos relativos, sólo definibles desde las consecuencias (Sánchez, 2016, p. 22).

El prefijo trans pareciera ser la clave de esta disolución requerida, porque de suyo implica movilidad, llevar de un sitio a otro, lo que va más allá o a través de, de tal suerte que Adame (2009) lo reivindica con la noción de un transujeto: el eterno caminante que funda y encuentra lugares o sitios en su transcurrir. La esencia nómada donde el recorrido se observa como acción estética, pero este errar no es algo estrictamente físico, sino que implica pensamiento, visión y contexto; el trans de la transparencia figurativa que nos reivindica como virtualidad y potencia, pero también como fantasma y memoria.

Lo que está en juego es el rompimiento de un paradigma, la crisis de una identidad, los modos de hacer y relacionar el pensamiento que conduce a una negación, puesto que no se parte de certezas. Con ello se declara que no todos los profesores avanzan sobre esta perspectiva, situación común que pone de manifiesto aspectos formativos, profesionales y de actitud. El proyecto obliga a tomar el riesgo de al menos ofrecer una posibilidad de cambio, puesto que apostar por la transdisciplinariedad implica reajustes y evolución constantes. Hemos elaborado así un proyecto que se teje sobre las bases mismas del dinamismo, por lo que se requiere del juego creativo:

El juego es el antídoto contra la brutalidad; y la representación basada en la provisionalidad, en la modestia en la aceptación de realidades consensuadas, uno de los modos de practicar el juego sin por ello renunciar a la experiencia (Sánchez, 2016, p. 20).

La lucha se establece en el seno de la individualidad y de los parámetros del 
arte institucional y clásico con el que la mayoría de los académicos hemos sido formados en algún momento. Ante la necesidad de mostrar resultados concretos y específicos en proyectos que se diseñan en yuxtaposición, la diversidad propia de una "naturaleza" consciente se abre paso a través de una adaptación constante: la adaptación reclamada como proceso creativo.

\section{A modo de conclusión}

A modo de conclusión queremos enfatizar lo problemático que resulta un cambio de paradigma, así como su aceptación e inserción, no sólo dentro de los cánones institucionales, sino de las estructuras mentales que conforman nuestra visión del mundo y del arte en particular. Romper con los patrones de una educación que ahora se evidencia multidimensional y multidisciplinaria conlleva el reto de un ser humano cooperativo que no impone su conocimiento o su experiencia, sino que los otorga. En este aspecto, el camino a recorrer es largo, por cuanto exige el sistema educativo, para establecer los parámetros requeridos en la evaluación y pertinencia. ¿Cómo medir, objetivar y validar un conocimiento que se desarrolla en procesos sistémicos y nace de la complejidad?, ¿cómo mantenerlo vigente en una visión que, por excelencia, nos permita acceder a la creación? Y en lo humano, ¿cómo ponderar los procesos creativos y adaptativos del hombre en sociedad para darnos el permiso de admitirlos como arte?

Quedan estas líneas que esperemos despierten el interés por una movilización de nuestras estructuras y como una invitación a la permanente búsqueda.

\section{Bibliografía}

AA.VV. (2005). Teoría del constructivismo social de Lev Vigotsky en comparación con la teoría Jean Piaget. En sitio web constructivismos.blogspot. Recuperado el 30 de julio de 2013 en http://constructivismos.blogspot.mx/

AA. VV. (2015). Afectos y saberes, crítica, cultura y desestabilización de la representación. México: Universidad Iberoamericana.

Adame, D. (2009). Conocimiento y representación: un re-aprendizaje hacia la transteatralidad. México: Universidad Veracruzana.

Araujo, G. y Fernández, L. (1999). La entrevista grupal: herramienta de la metodología cualitativa de investigación. En Szasz, I. y Lerner, S. Para comprender la subjetividad. Investigación cualitativa en salud retrospectiva y sexualidad. Ciudad de México: Colegio de México.

Beuchot, M. (2002). Estudios sobre Peirce y la Escolástica. Disponible en http://dadun. unav.edu/bitstream/10171/5881/1/150.pdf

Díaz Barriga, F. et al. (1990). Metodología de diseño curricular para la educación superior. México: Trillas.

Díaz Barriga, A. (1996). Ensayos sobre la problemática curricular. México: Trillas.

Espina, M. (2004). Complejidad y pensamiento social. En Transdisciplinariedad y Complejidad en el Análisis Social. Gestión de las Transformaciones Sociales, MOST, documento de debate $n^{\circ}$ 70, UNESCO. 9-28. Recuperado de http://unesdoc.unesco.org/images/0013/001363/136367s.pdf 
Heidegger, M. (1997). Filosofía, ciencia y técnica. Chile: Universitaria Editorial.

Modelo Universitario Minerva (MUM). (2007). Fundamentos. Benemérita Universidad Autónoma Puebla. México.

Modelo Universitario Minerva (MUM). (2007). Modelo Educativo Académico. Benemérita Universidad Autónoma Puebla. México.

Montecinos H. (2011). El humanismo según la filosofía. En sitio web Filosofía.mx. Recuperado el 27 de agosto de 2013 en

http://www.filosofia.mx/index.php?/forolibre/archivos/el_humanismo_segun_la_filosofia

Morin, E. (2014). El método 5. La humanidad de la humanidad. La identidad humana. Madrid, España: Cátedra.

Morin, E. (2010). El método 3. El conocimiento del conocimiento. Madrid, España: Cátedra.

Nicolescu, B. (1996). La transdisciplinariedad. Manifiesto. Ciudad de México, México: 7 Saberes.

Ruiz Ruiz, J. M. (2005). Teoría del currículum: diseño, desarrollo e innovación curricular. Madrid: Universitas.

Sánchez A. J. (2013). Prácticas de lo real en la escena contemporánea. México: Paso de Gato.

Sánchez A. J. (2016). Ética y representación. México: Paso de Gato.

Sánchez A. J y Belvis E. (2015). No hay más poesía que la acción. México: Paso de Gato.

Valles R. (2015). Teatro Antilógico. México: Paso de Gato.

Vattimo, G. et, al. (2007). El sentido de la existencia. Posmodernidad y nihilismo. Bilbao, Portugal: Universidad de Deusto.

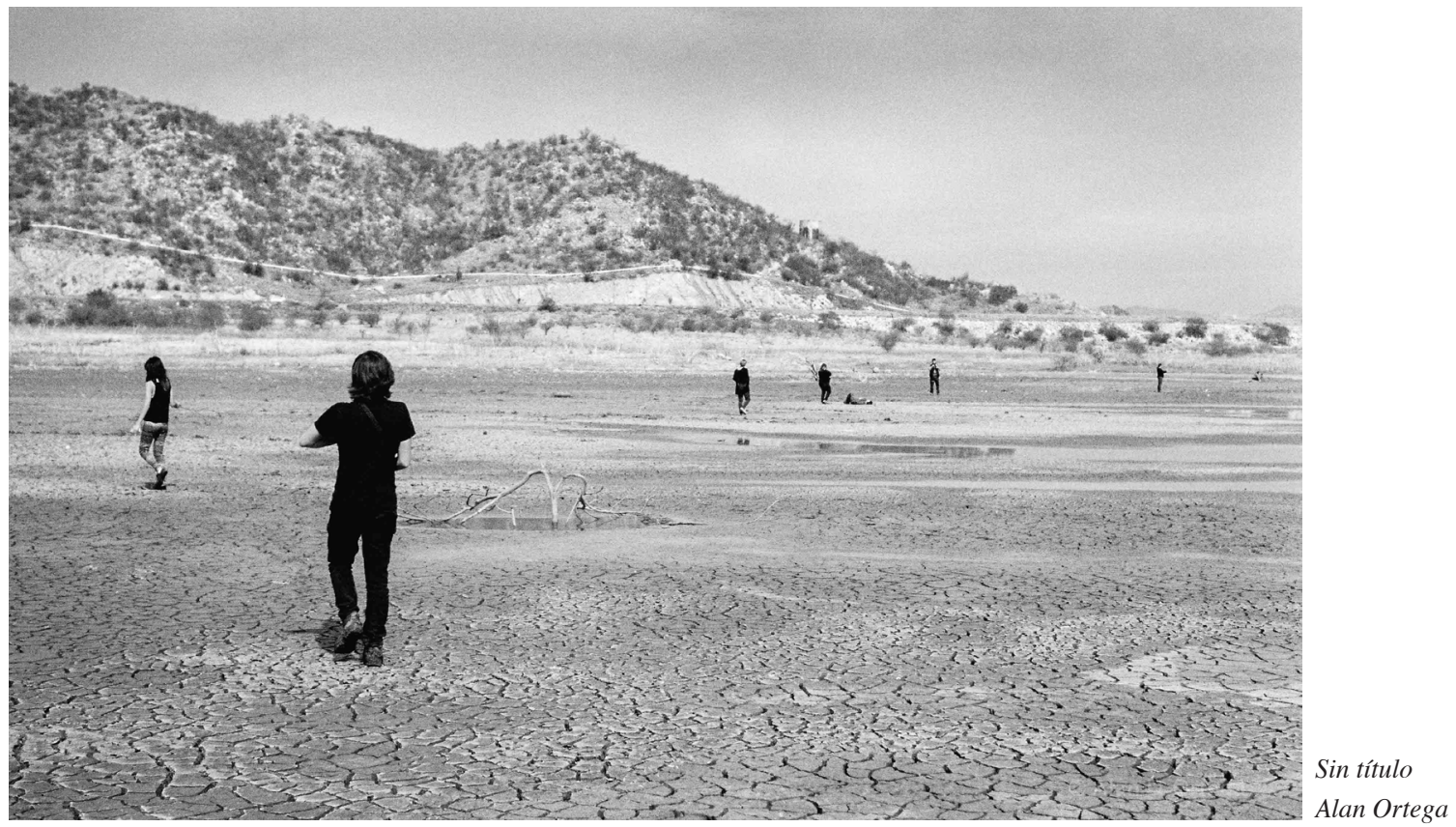

